\title{
Carotenoids as biomarkers of fruit and vegetable intake in men and women
}

\author{
Charles Couillard ${ }^{1,2 *}$, Simone Lemieux ${ }^{1,2}$, Marie-Claude Vohl ${ }^{1,2}$, Patrick Couture ${ }^{2,3}$ and Benoit Lamarche La $^{1,2}$ \\ ${ }^{1}$ School of Nutrition, Université Laval, Québec, Canada, G1V OA6 \\ ${ }^{2}$ Institute of Nutrition and Functional Foods, Université Laval, Québec, Canada, G1V OA6 \\ ${ }^{3}$ Lipid Research Center, Centre hospitalier universitaire de Québec (CHUL), Québec, Canada, G1V $4 G 2$
}

(Submitted 12 April 2016 - Final revision received 8 July 2016 - Accepted 27 July 2016 - First published online 30 August 2016)

\section{Abstract}

High fruit and vegetable (FAV) intake is associated with a lower prevalence of chronic diseases. Identifying the ideal number of FAV servings needed to reduce chronic disease risk is, however, difficult because of biases inherent to common self-report dietary assessment tools. The aim of our study was to examine the associations between daily FAV intake and plasma carotenoid concentrations in men and women enrolled in a series of fully controlled dietary interventions. We compiled and analysed data from a group of 155 men and 109 women who participated in six fully controlled dietary interventions and compared post-intervention fasting plasma carotenoid ( $\alpha$-carotene, $\beta$-carotene, $\beta$-cryptoxanthin, lutein, lycopene, zeaxanthin) concentrations with regard to the daily FAV servings consumed by the participants. We found that plasma $\beta$-cryptoxanthin, lutein and zeaxanthin concentrations were positively associated with daily FAV servings $(P \leq 0 \cdot 005)$. However, daily FAV intake was negatively associated with plasma $\alpha$-carotene $(P<0 \cdot 0005)$ and lycopene $(P<0 \cdot 0001)$ concentrations, whereas no association was noted with plasma $\beta$-carotene. When men and women were analysed separately, we found that for any given number of FAV servings consumed women had higher circulating lutein concentrations compared with men $(P<0 \cdot 01)$. Significant sex $\times$ FAV $(P<0 \cdot 0001)$ and sex $\times$ dietary $\beta$-cryptoxanthin $(P<0.0005)$ interactions were also noted favouring higher plasma $\beta$-cryptoxanthin concentrations in women than in men for a given FAV consumption. Results from these fully controlled dietary feeding studies indicate that plasma $\beta$-cryptoxanthin and lutein concentrations can be used as robust biomarkers of FAV consumption. They also suggest the existence of sex differences influencing circulating $\beta$-cryptoxanthin and lutein concentrations following FAV consumption.

Key words: Carotenoids: Fruit and vegetable intake: Sex differences: Diet-controlled intervention studies: Biomarkers

Healthy nutritional habits are associated with a reduced risk of chronic diseases, and most nutritional guidelines advocate increasing the consumption of fruits and vegetables (FAV) in order to prevent CVD, type 2 diabetes (T2D) and some types of cancer ${ }^{(1-4)}$. The extent of health benefits resulting from increased FAV consumption, however, depends on the adherence of individuals to prescribed nutritional recommendations. Many methods are commonly used to evaluate food consumption, including food diaries, FFQ and 24-h recalls ${ }^{(5)}$. Automated forms of these tools are particularly affordable, can be easily administered and provide proper estimates of food and nutrient intakes, which explain their use in large-scale clinical trials and epidemiological studies ${ }^{(5)}$. However, these methods also rely on self-report, which has been heavily criticised recently ${ }^{(6)}$, with potentially important estimation biases regarding certain foods ${ }^{(7-9)}$.

Measuring markers of food consumption in various biological specimens (urine, blood, tissues, etc.) has therefore been suggested to be a more accurate and consistent indication of dietary intakes and patterns ${ }^{(10)}$. To that effect, recovery biomarkers (e.g. doubly labelled water, urinary measures) are more accurate in estimating dietary intakes compared with concentration biomarkers such as circulating carotenoids, which are, however, less expensive and often easier to collect. Carotenoids are a family of more than 700 fat-soluble pigments that are present in varying quantities in $\mathrm{FAV}^{(11)}$. As they cannot be synthesised by humans ${ }^{(10)}$, carotenoids found in circulation are considered to be reliable biomarkers of dietary carotenoid intake, even though conditions such as obesity have been shown to influence this association ${ }^{(12)}$. Accordingly, FAV consumption accounts for up to $90 \%$ of dietary carotenoid intake with the most common blood carotenoids being $\alpha$-carotene, $\beta$-carotene, $\beta$-cryptoxanthin, lutein, lycopene and zeaxanthin, which represent $>95 \%$ of total circulating carotenoids $^{(11)}$.

The use of circulating carotenoid concentrations as biomarkers of FAV intake has been mostly validated against self-reported measures of FAV consumption ${ }^{(13-18)}$. On the other hand, fully controlled dietary interventions - that is, studies in which subjects are provided with all the foods to consume

Abbreviation: FAV, fruits and vegetables.

* Corresponding author: C. Couillard, email charles.couillard@fsaa.ulaval.ca 
and do so under the supervision of research staff - provide a more robust setting to assess the concordance between blood concentrations of biomarkers and food intake. Over the past few years, we have conducted a series of fully controlled dietary interventions with a wide range of daily FAV servings ${ }^{(19-23)}$. The present analysis was therefore conducted with the intent to examine the extent to which circulating carotenoid concentrations are concordant with variations in dietary carotenoid and FAV intakes of healthy men and women enrolled in fully controlled dietary interventions. We hypothesise that dietary carotenoid and FAV intakes are positively associated with plasma carotenoid concentrations in both men and women.

\section{Methods}

\section{Study population}

A total of 155 men and 109 women have participated in different fully controlled dietary interventions undertaken in our research facility since 2004. Specific selection criteria for participants of each intervention have been previously published $^{(19-23)}$. Overall, participants had to be healthy, and were excluded on the basis of current treatment for history of hypertension, CVD, T2D or other endocrine disorders, if they were smokers or had an alcohol consumption $>1$ drink/d. Dietary supplement use was also considered a reason to exclude participants from all the studies. Each participant signed a written informed consent from to take part in the studies, which were all approved by the Human Research Ethics Committee of Université Laval.

\section{Description of fully controlled dietary interventions}

As indicated above, we have taken advantage of data from a series of isoenergetic fully controlled dietary interventions conducted over the last 10 years by our research group ${ }^{(19-23)}$ in order to perform the present analysis. Complete detailed descriptions of diet 1 (registered at ClinicalTrials.gov as NCT00988650) $)^{(20)}$, diet 2 (NCT00930137) $)^{(23)}$, diet 3 (NCT013$51012)^{(21)}$, diet 4 (NCT01163175) $^{(22)}$, diet 5 (NCT01293344 $^{(19)}$ and diet 6 (NCT00988650) ${ }^{(20)}$ have been previously published. In brief, diet 1 (6.8 (SD 1.0) FAV servings/d) was a 5-week control diet of a study assessing the impact of the Mediterranean diet and was designed to reflect average macronutrient intakes of North American men ${ }^{(24)}$; diets 2 (7.4 (SD 0.9) FAV servings/d) and 4 (9.8 (sD 1.6) FAV servings/d) were 4-week control diets low in trans-fatty acids (TFA) of ruminant origin ( 0.3 and $0.8 \%$ of energy, respectively) from studies investigating the metabolic impact of TFA from milk; diet 3 (9.6 (sD 2.4) FAV servings/d) was part of a study looking at the metabolic impacts of different vegetable oils and consisted of a 4-week, weightmaintaining diet of fixed macronutrient composition for protein ( $15 \%$ of energy), carbohydrates (50\% of energy) and fats (35\% of energy), with a blend of maize and safflower oils (25:75) contributing to dietary fat intake (18\%); diets 5 (19.1 (SD 3.6) FAV servings/d) and 6 (21.0 (sD 3.0) FAV servings/d) corresponded to 5 -week Mediterranean diets - that is, they contained key foods of the Mediterranean pyramid (e.g. olive oil, whole-grain products, FAV, legumes, nuts, cheese and yogurt, fish, poultry, red wine) $)^{(25)}$ and provided a MUFA:SFA ratio of 2.7 corresponding to the one found in the traditional Mediterranean diet ${ }^{(26)}$. Table 1 summarises the macronutrient composition of the different dietary interventions, whereas the online Supplementary Tables S1 and S2 list the FAV provided and consumed by the participants in each diet.

In all cases, subjects arrived at our Clinical Investigation Unit (CIU) on weekdays to have their body weight measured and consume their lunch meal (approximately 35\% of daily energy intake) under staff supervision. All meals (including foods and beverages) were prepared and packaged at the CIU, and participants were provided with their evening meals and the next day's breakfast to take home, giving us the opportunity to control for energy, macronutrient and food intakes. Food was provided under isoenergetic conditions in order to maintain body weight constant, and a 7-d cyclic menu was used in all dietary interventions. Participants were instructed to consume only the foods provided. Compliance of participants with their specific diets was excellent (>95\% in all cases). Dietary interventions were formulated using the Nutrition Data System software (version 4.03_31; Nutrition Coordinating Center). Participants were instructed to maintain their usual physical activity level throughout the experiments except for the $3 \mathrm{~d}$ that preceded blood sampling at the end of the feeding period, during which they were asked to refrain from intense physical exercise. Dietary carotenoid intake was calculated using the Canadian Nutrient File (http://webprod3.hc-sc.gc.ca/cnf-fce/ index-eng.jsp), which was also used to obtain the energy, macronutrient and micronutrient contents of the different diets.

\section{Anthropometry and plasma lipids}

Body weight, height as well as waist and hip circumferences of each participant were measured by standardised procedures ${ }^{(27)}$.

\section{Plasma carotenoids}

Chemicals. Retinyl acetate, $\alpha$-carotene, lutein, zeaxanthin, $\beta$-cryptoxanthin, $\beta$-carotene and lycopene were purchased from Sigma. All solvents were of HPLC grade and purchased from VWR. HPLC water was obtained using a MilliQ water purification system from Millipore.

Standards preparation. Stock solutions for each carotenoid were prepared ( $1 \mathrm{mg}$ in $100 \mathrm{ml}$ of solvent) in either ethanol $\left(\mathrm{C}_{2} \mathrm{H}_{6} \mathrm{O}\right.$, for lutein, zeaxanthin and $\beta$-cryptoxanthin) or hexane $\left(\mathrm{C}_{6} \mathrm{H}_{14}\right.$, for $\beta$-carotene and lycopene). Solutions were left to shake overnight at $4^{\circ} \mathrm{C}$ under dim light. The exact concentration of each stock solution was then determined using a UV spectrophotometer, and the specific molecular extinction coefficient (e) of each carotenoid was determined ${ }^{(28)}$. Appropriate volumes of stock solutions were then transferred to amber Eppendorf tubes and evaporated under N. On the day of the analyses, carotenoid standards were solubilised with 
methanol-dichloromethane $(65: 35, \mathrm{v} / \mathrm{v})$ to obtain a final concentration of $2 \mu \mathrm{m}$. These solutions were then diluted to obtain calibration curves. Retinyl acetate $(15 \mu \mathrm{m})$ was used as an internal standard.

Samples preparation. Post-intervention plasma samples maintained at $-80^{\circ} \mathrm{C}$ were thawed a day before analyses. Samples were vortexed and then centrifuged at $3500 \mathrm{rpm}$ for $10 \mathrm{~min}$ at $4^{\circ} \mathrm{C}$. Aliquots of $100 \mu \mathrm{l}$ of plasma were then transferred to Eppendorf tubes $(1.5 \mathrm{ml})$ along with $20 \mu \mathrm{l}$ of 2-propanol and $20 \mu \mathrm{l}$ of carotenoid standard, and the tubes were vortexed. Samples were transferred to a $400-\mu \mathrm{l}$ fixed well plate (ISOLUTE ${ }^{\circledR}$ SLE+; Biotage), and $900 \mu$ l of hexaneisopropanol (90:10, v/v) was added to each well. Each extracted sample was evaporated under $\mathrm{N}$, and once dry the sample was reconstituted with $300 \mu \mathrm{l}$ of methanoldichloromethane $(65: 35, \mathrm{v} / \mathrm{v})$. Plates were shaken for $10 \mathrm{~min}$, and samples were transferred into HPLC glass vials to be analysed.

HPLC. HPLC-UV analysis of the samples was performed using an Agilent 1260 liquid handling system (Agilent) equipped with a binary pump system and a C30 reversed phase column (YMC America Inc.) kept at constant temperature $\left(35^{\circ} \mathrm{C}\right)$. Carotenoids of the different samples were separated with a mobile phase consisting of methanol-water (98:2, v/v; Eluent A) and methyltert-butyl ether (Eluent B; VWR). Flow rate was set at $1 \mathrm{ml} / \mathrm{min}$, and the gradient elution was as follows: $2 \%$ Eluent B (initial), $2 \cdot 0-80 \%$ Eluent B $(0 \cdot 0-27.0 \mathrm{~min})$, isocratic $80 \%$ Eluent B (27.0-31.0 min), $80 \cdot 0-2 \cdot 0 \%$ Eluent $B \quad(31 \cdot 0-31 \cdot 1 \mathrm{~min})$ and isocratic $2 \%$ Eluent B (31.1-34.0 min). The UV detector was set at $450 \mathrm{~nm}$, and identification of each compound was confirmed using retention time and UV spectra $(190-640 \mathrm{~nm})$ of the pure compounds. Data acquisition was carried out using Chemstation software (Agilent).

\section{Statistical analyses}

Data are presented as mean values and standard deviations unless stated otherwise. General linear model (GLM) procedures were used to test differences in the dietary composition as well as plasma carotenoid concentrations of participants enrolled in the different nutritional interventions. Associations of daily servings of FAV and dietary carotenoid intake estimated from the nutrient files with post-intervention plasma carotenoid concentrations were tested for linear trend using the GLM procedure with statistical adjustment for sex and age, when appropriate. The interaction between sex and FAV intake in determining the blood concentrations of the various carotenoids was investigated using proper interaction terms in the GLM. Spearman's correlation coefficients were computed to study the associations between body weight and total carotenoid dietary intake and plasma concentrations. All analyses were performed using SAS statistical package (version 9.3, SAS Institute Inc.). Throughout the analyses, a $P \leq 0.05$ was considered to be statistically significant.

\section{Results}

Physical characteristics as well as FAV and dietary carotenoid intakes of participants enrolled in different dietary interventions sorted according to the daily number of FAV servings consumed are presented in Table 1. There were significant differences in physical characteristics between diet groups due to variations in the specific selection criteria of the six diet-controlled interventions that we used in this analysis. The mean age of the 264 participants was 42.6 (SD 14.4) years, $41 \%$ of them were women, and interventions lasted between 4 and 5 weeks. Daily FAV intake of the different diets averaged $12 \cdot 3$ (sD 5.8) servings and ranged from 6.8 (SD 1.0) to 21.0 (SD 3.0) servings/d $(P<0.0001$ for linear trend; Table 2). Similar trends were observed when FAV servings were expressed per a standard 2500 -kcal energy intake or in $\mathrm{g} / \mathrm{d}(P<0.0001)$. We found that the increment in daily FAV servings across diets was attributable to increases in both fruit $(P<0 \cdot 0001)$ and vegetable servings $(P<0.0001)$, with the number of daily vegetable servings consumed by the participants remaining slightly above than that of fruits in all diets. Furthermore, we found positive linear trends for dietary $\alpha$-carotene, $\beta$-carotene, $\beta$-cryptoxanthin and lutein intakes $(P<0.0001$ for all; Table 2$)$, whereas there were significant negative linear trends $(P<0.0001)$ for dietary lycopene and retinol intakes when diets were ranked according to daily FAV servings consumed.

Fig. 1 illustrates the linear trend analysis of the associations of plasma $\alpha$-carotene, $\beta$-carotene, lycopene and retinol concentrations with their corresponding dietary intakes as well as with the daily number of FAV servings consumed by the participants. Circulating concentrations of $\alpha$-carotene and lycopene were positively associated with their corresponding dietary intakes $(P<0.0001$ for linear trend for both) but negatively with the number of FAV consumed daily $(P<0.0001$ for linear trend for both). Plasma $\beta$-carotene and retinol concentrations showed no significant association with either their daily dietary intakes or number of FAV servings consumed (Fig. 1).

As illustrated in Fig. 2, we found that plasma $\beta$-cryptoxanthin, lutein and zeaxanthin concentrations were all positively associated with their corresponding dietary intakes $(P \leq 0.0005)$ as well as with the daily number of FAV servings consumed $(P \leq 0.005)$. Similar patterns of associations were noted when we considered daily fruit or vegetable servings separately (data not shown).

As lutein and $\beta$-cryptoxanthin were found to be the best correlates of daily FAV servings consumed, we compared the associations of plasma lutein and $\beta$-cryptoxanthin concentrations with their respective daily dietary intakes and FAV servings consumed in men and women separately (Fig. 3). We found significant sex $\times$ dietary $\beta$-cryptoxanthin $(P<0.0005)$ and sex $\times$ FAV servings $(P<0.005)$ interactions with regard to circulating $\beta$-cryptoxanthin concentrations, as women showed a steeper increase in plasma $\beta$-cryptoxanthin concentrations with increasing dietary $\beta$-cryptoxanthin intake and daily number of FAV servings consumed compared with men (Fig. 3). In contrast, significantly higher plasma lutein concentrations were noted in women at any concentration of dietary lutein + zeaxanthin intake or daily number of FAV 
Table 1. Characteristics of the participants and macronutrient content of the different diets*

(Mean values and standard deviations)

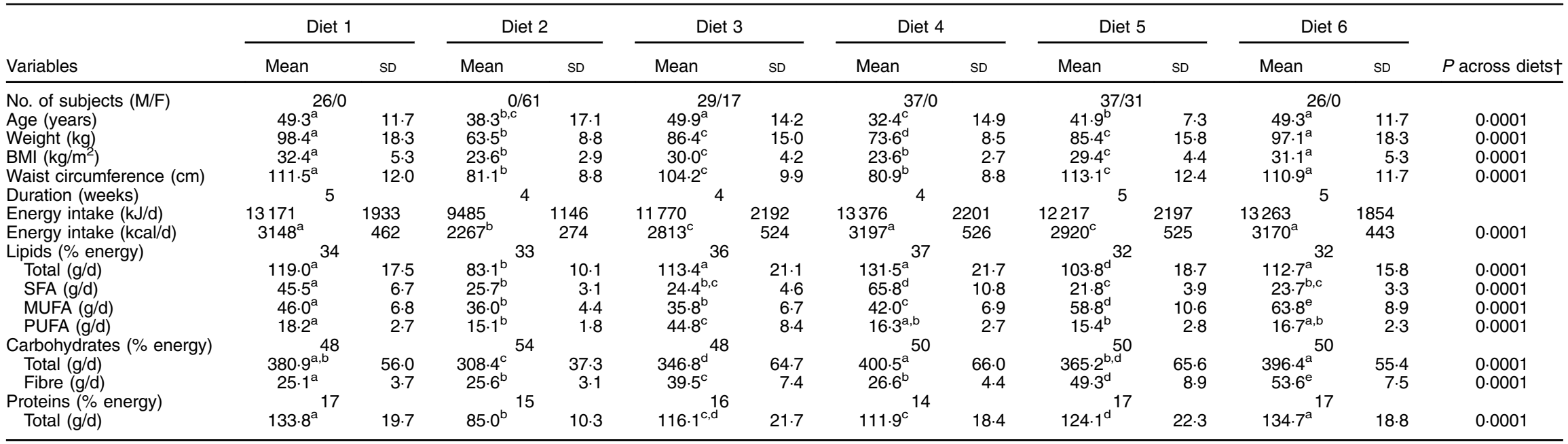

$\mathrm{M} / F$, male/female.

values with unlike superscript letters were significantly different.

* Macronutrients reported as percentage of daily energy have no standard deviations because all subjects consumed the same diet; hence, there is no between-subject variation in these variables except for the mean of all six diets. t Data were adjusted for sex and age.

Table 2. Characteristics and daily micronutrient intakes of participants

(Mean values and standard deviations)

\begin{tabular}{|c|c|c|c|c|c|c|c|c|c|c|c|c|c|}
\hline \multirow[b]{2}{*}{ Variables } & \multicolumn{2}{|c|}{ Diet 1} & \multicolumn{2}{|c|}{ Diet 2} & \multicolumn{2}{|c|}{ Diet 3} & \multicolumn{2}{|c|}{ Diet 4} & \multicolumn{2}{|c|}{ Diet 5} & \multicolumn{2}{|c|}{ Diet 6} & \multirow[b]{2}{*}{$P$ across diets ${ }^{*}$} \\
\hline & Mean & SD & Mean & SD & Mean & SD & Mean & SD & Mean & SD & Mean & SD & \\
\hline FAV (servings/d) & $6 \cdot 8^{\mathrm{a}}$ & 1.0 & $7 \cdot 4^{\mathrm{a}}$ & 0.9 & $9 \cdot 6^{\mathrm{b}}$ & $2 \cdot 4$ & $9.8^{\mathrm{b}}$ & 1.6 & $19 \cdot 1^{c}$ & 3.6 & $21 \cdot 0^{\mathrm{d}}$ & 3.0 & 0.0001 \\
\hline FAV (servings $/ \mathrm{d}$ per $10460 \mathrm{~kJ}(2500 \mathrm{kcal}))$ & $5 \cdot 4^{\mathrm{a}}$ & 0.1 & $8 \cdot 1^{\mathrm{b}}$ & 0.3 & $8 \cdot 4^{\mathrm{C}}$ & 0.6 & $7.7^{\mathrm{c}}$ & 0.2 & $16 \cdot 4^{\mathrm{d}}$ & 0.5 & $16 \cdot 6^{\mathrm{e}}$ & 0.3 & 0.0001 \\
\hline FAV $(g / d)$ & $652^{\mathrm{a}}$ & 96 & $627^{\mathrm{a}}$ & 80 & $761^{\mathrm{b}}$ & 187 & $909^{c}$ & 153 & $1431^{d}$ & 267 & $1569^{e}$ & 222 & 0.0001 \\
\hline Fruits (servings/d) & $1.9^{\mathrm{a}}$ & 0.3 & $2 \cdot 7^{\mathrm{b}}$ & 0.3 & $4 \cdot 7^{\mathrm{c}}$ & $1 \cdot 1$ & $3.0^{\mathrm{b}}$ & 0.5 & $7 \cdot 5^{\mathrm{d}}$ & 1.4 & $8.3^{\mathrm{e}}$ & $1 \cdot 2$ & 0.0001 \\
\hline Fruits (servings/d per $10460 \mathrm{~kJ}(2500 \mathrm{kcal}))$ & $1.6^{\mathrm{a}}$ & 0.4 & $2 \cdot 9^{\mathrm{b}}$ & 0.1 & $4 \cdot 1^{\mathrm{b}}$ & 0.3 & $2 \cdot 3^{\mathrm{c}}$ & 0.1 & $6 \cdot 4^{\mathrm{d}}$ & 0.2 & $6 \cdot 5^{\mathrm{e}}$ & 0.1 & 0.0001 \\
\hline Fruits (g/d) & $252^{\mathrm{a}}$ & 37 & $289^{\mathrm{a}}$ & 37 & $421^{\mathrm{b}}$ & 103 & $366^{c}$ & 62 & $646^{\mathrm{d}}$ & 121 & $708^{\mathrm{e}}$ & 100 & 0.0001 \\
\hline Vegetables (servings/d) & $4 \cdot 9^{\mathrm{a}}$ & 0.7 & $4 \cdot 7^{\mathrm{a}}$ & 0.6 & $4.9^{\mathrm{a}}$ & 1.2 & $6 \cdot 8^{\mathrm{b}}$ & $1 \cdot 1$ & $11.6^{\mathrm{c}}$ & $2 \cdot 2$ & $12 \cdot 7^{\mathrm{d}}$ & 1.8 & 0.0001 \\
\hline Vegetables (servings $/ \mathrm{d}$ per $10460 \mathrm{~kJ}(2500 \mathrm{kcal}))$ & $3.9^{\mathrm{a}}$ & 0.1 & $5 \cdot 2^{\mathrm{b}}$ & 0.2 & $4 \cdot 3^{\mathrm{c}}$ & 0.3 & $5 \cdot 3^{\mathrm{d}}$ & 0.1 & $9.9^{\mathrm{e}}$ & 0.3 & $10 \cdot 1^{\dagger}$ & 1.2 & 0.0001 \\
\hline Vegetables $(\mathrm{g} / \mathrm{d})$ & $400^{\mathrm{a}}$ & 59 & $338^{\mathrm{b}}$ & 43 & $340^{\mathrm{b}}$ & 84 & $543^{\mathrm{C}}$ & 91 & $785^{\mathrm{d}}$ & 147 & $861^{\mathrm{e}}$ & 122 & 0.0001 \\
\hline$a$-Carotene $(\mathrm{mg} / \mathrm{d})$ & $1.64^{\mathrm{a}}$ & 0.24 & $1.26^{\mathrm{b}}$ & 0.15 & $2 \cdot 15^{\mathrm{c}}$ & 0.40 & $4 \cdot 25^{d}$ & 0.70 & $1 \cdot 81^{\mathrm{a}, \mathrm{e}}$ & 0.33 & $1.96^{\mathrm{e}}$ & 0.28 & 0.0001 \\
\hline$\beta$-Carotene $(\mathrm{mg} / \mathrm{d})$ & $5 \cdot 67^{\mathrm{a}}$ & 0.83 & $4.52^{\mathrm{b}}$ & 0.55 & $11 \cdot 10^{c}$ & 2.07 & $10 \cdot 91^{c, d}$ & 1.80 & $9.51^{\mathrm{e}}$ & 1.71 & $10 \cdot 33^{d}$ & 1.44 & 0.0001 \\
\hline$\beta$-Cryptoxanthin (mg/d) & $0.66^{a}$ & 0.10 & $0.55^{\mathrm{b}}$ & 0.07 & $0.23^{c}$ & 0.04 & $0.24^{c}$ & 0.04 & $1.65^{\mathrm{d}}$ & 0.30 & $1.79^{\mathrm{e}}$ & 0.25 & 0.0001 \\
\hline Lutein + zeaxanthin $(\mathrm{mg} / \mathrm{d})$ & $3 \cdot 19^{\mathrm{a}}$ & 0.47 & $2 \cdot 62^{\mathrm{b}}$ & 0.32 & $5 \cdot 55^{\mathrm{c}}$ & 1.04 & $3.45^{\mathrm{a}}$ & 0.57 & $6 \cdot 20^{d}$ & $1 \cdot 11$ & $6.73^{\mathrm{e}}$ & 0.94 & 0.0001 \\
\hline Lycopene $(\mathrm{mg} / \mathrm{d})$ & $18 \cdot 25^{\mathrm{a}}$ & 2.68 & $8 \cdot 67^{\mathrm{b}}$ & 1.05 & $6 \cdot 46^{c}$ & 1.21 & $18 \cdot 46^{\mathrm{a}}$ & 3.04 & $9 \cdot 39^{b, d}$ & 1.69 & $10 \cdot 20^{d}$ & 1.43 & 0.0001 \\
\hline Retinol (mg/d) & $0.69^{a}$ & 0.10 & $0.41^{b}$ & 0.05 & $0.43^{\mathrm{b}}$ & 0.08 & $1 \cdot 19^{c}$ & 0.20 & $0.29^{d}$ & 0.05 & $0.32^{d}$ & 0.04 & 0.0001 \\
\hline
\end{tabular}

FAV, fruits and vegetables.

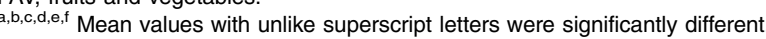

* Data were adjusted for sex and age. 

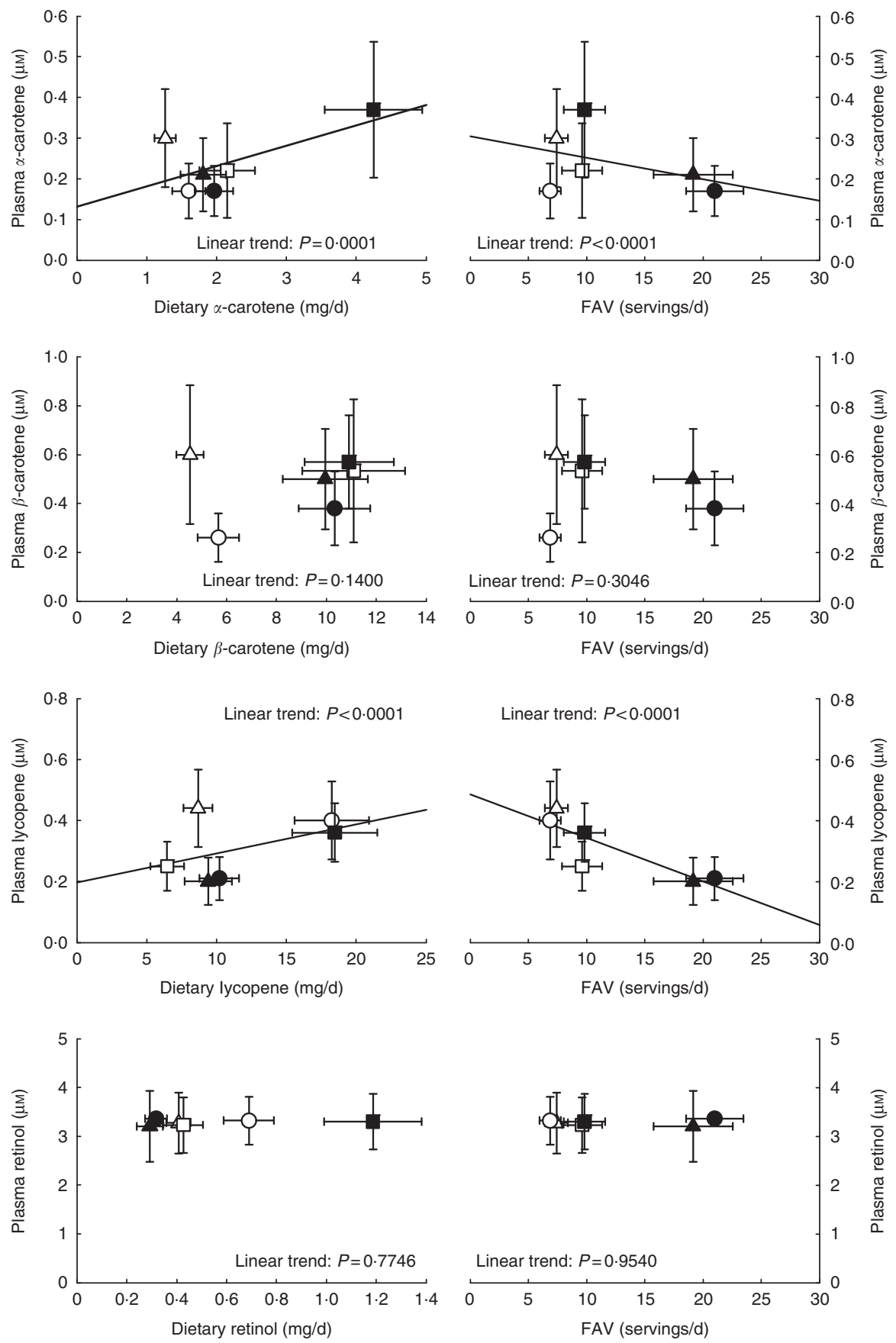

Fig. 1. Mean plasma $\alpha$-carotene, $\beta$-carotene, lycopene and retinol concentrations of participants according to their respective mean daily dietary intake as well as daily fruit and vegetable (FAV) consumption. Values are means and standard deviations. Data were adjusted for sex and age. $\bigcirc$, Diet 1; $\triangle$, diet 2; $\square$, diet 3; $\square$, diet 4; $\boldsymbol{\Delta}, \operatorname{diet} 5 ; \mathbf{0}, \operatorname{diet} 6$.

servings compared with men (sex effect: $P<0.0001$ for both). However, no significant interaction was noted between dietary lutein + zeaxanthin intake or daily number of FAV servings consumed and circulating lutein concentrations (Fig. 3). Similar results were obtained when plasma zeaxanthin concentrations were analysed alone (data not shown).

Finally, we looked at potential contributors to the variations in circulating carotenoid concentrations (Fig. 4), and found a significant positive association between BMI of participants and total dietary carotenoid intake $(r \quad 0.28, \quad P<0.0001)$. In contrast, BMI was negatively correlated to circulating carotenoid concentrations $(r-0 \cdot 33, P<0 \cdot 0001)$

\section{Discussion}

Results of the present analyses of data from highly controlled feeding studies provide further support to the use of plasma 

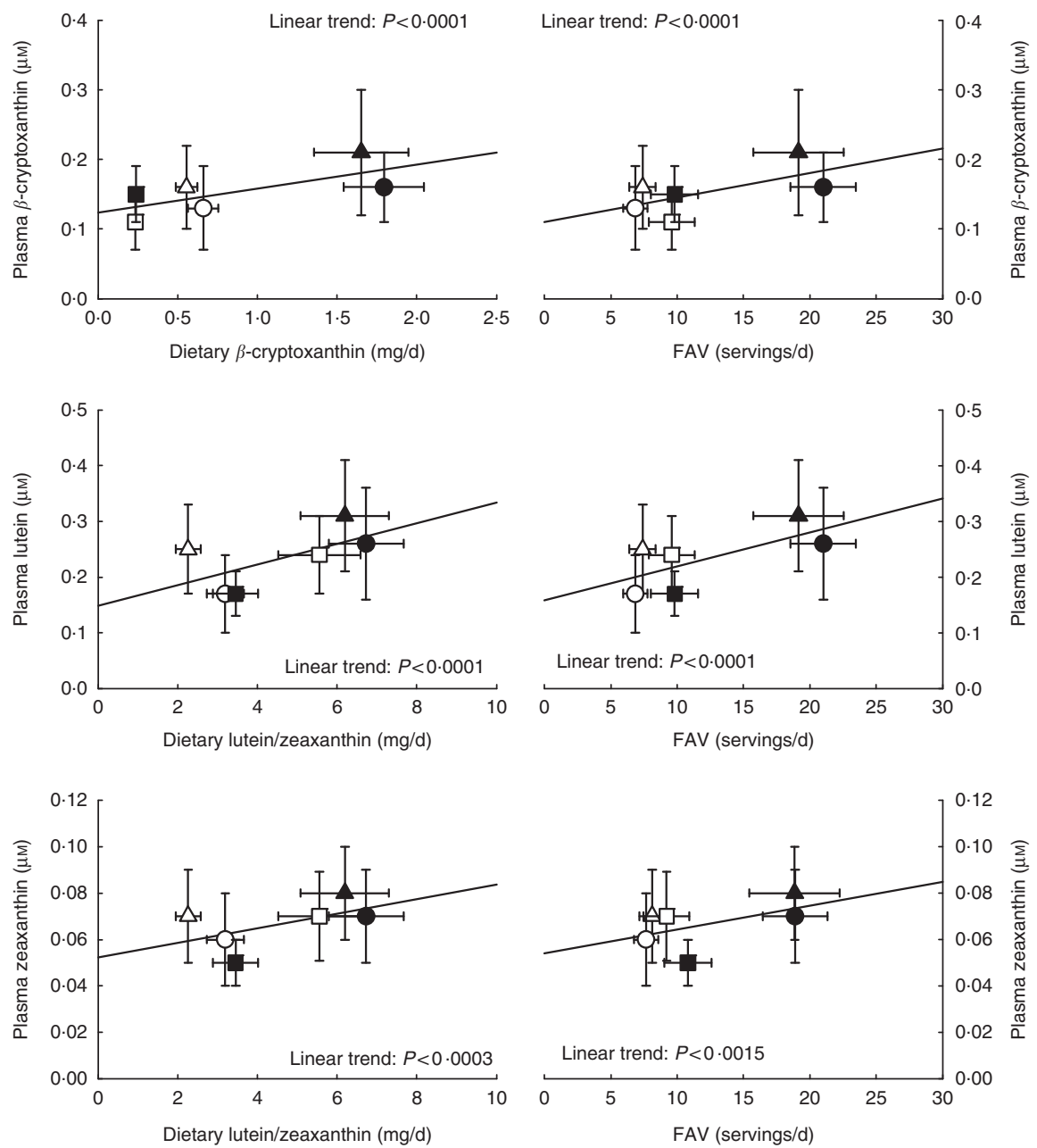

Fig. 2. Mean plasma $\beta$-cryptoxanthin, lutein and zeaxanthin concentrations of participants according to their respective mean daily dietary intake as well as fruit and vegetable (FAV) consumption. Values are means and standard deviations. Data were adjusted for sex and age. $\bigcirc$, Diet 1 ; $\triangle$, diet 2 ; $\square$, diet 3 ;, diet $4 ; \mathbf{\Delta}$, diet 5 ; , diet 6.

$\beta$-cryptoxanthin and lutein concentrations as biomarkers of mixed FAV consumption in both men and women. Our observations are in line with previous results, mostly based on self-report data, showing that circulating $\beta$-cryptoxanthin

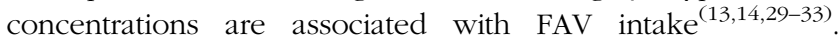
Furthermore, there is a large body of evidence showing that plasma lutein concentrations are also associated with FAV intake ${ }^{(13,29,32,34,35)}$. We also found that plasma zeaxanthin concentrations were positively associated with FAV intake, but their circulating concentrations remained 2-3 times lower than those of $\beta$-cryptoxanthin and lutein. However, contrary to what we observed, plasma $\beta$-cryptoxanthin concentrations of men and women were unchanged following increased daily FAV consumption (from 2 to 5 servings/d) for a period of $14 \mathrm{~d}^{(34)}$, but the authors were unable to offer a reasonable explanation for the lack of response of plasma $\beta$-cryptoxanthin to a higher FAV intake. The lack of association of circulating lutein concentrations with FAV intake in postmenopausal women has also been reported ${ }^{(30)}$.

On the other hand, although FAV are the primary source of dietary carotenoids, our analyses indicate that plasma $\alpha$-carotene concentrations are negatively associated with the daily number of FAV servings consumed by the participants enrolled in these diet-controlled feeding studies, whereas plasma $\beta$-carotene concentrations were not associated with FAV intake. This is somewhat contradictory to previous self-report data studies that have shown positive associations between circulating $\alpha$-carotene and $\beta$-carotene concentrations with vegetable ${ }^{(14,32,36,37)}$ and fruit ${ }^{(13,14,34)}$ consumption. A likely explanation for the inverse relationship between plasma $\alpha$-carotene concentration and FAV intake could be that, although FAV intake of diet 4 was in the lower range of intakes in studies we analysed, it was also the one showing the highest proportion of carrots (approximately $12 \%$ of total FAV intake or 1.25 servings/d), the most potent dietary source of $\alpha$-carotene ${ }^{(11)}$. This may have increased circulating $\alpha$-carotene concentrations of participants enrolled in diet 4 in the context of a lower FAV intake. Likewise, a high proportion of $\beta$-carotene-rich foods ${ }^{(11)}$ in diets with a lower FAV intake, such as green leafy vegetables in diet 3 (>18\% of total FAV intake or 1.76 servings/d) and butter $(75 \mathrm{~g} / 10460 \mathrm{~kJ}$ $(2500 \mathrm{kcal})$ ) in diet 4 , may have prevented us from noticing the previously reported association between plasma $\beta$-carotene 

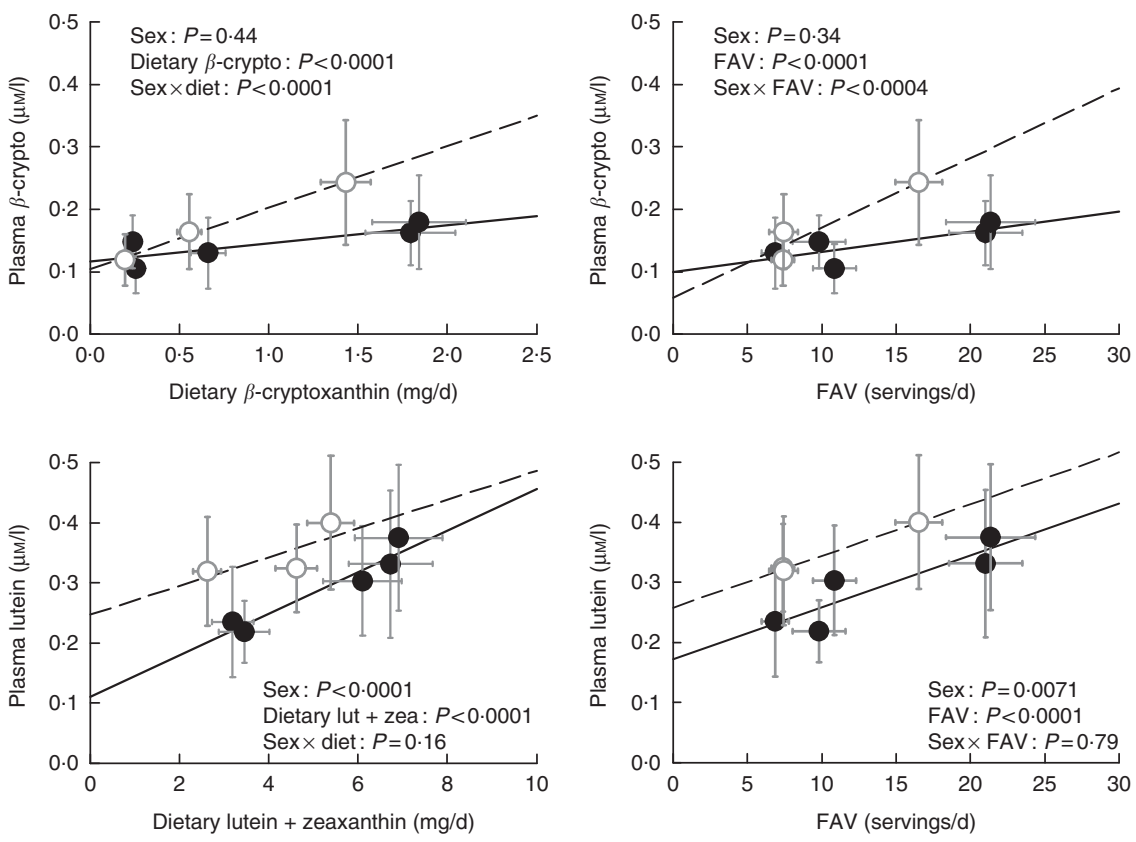

Fig. 3. Mean plasma $\beta$-cryptoxanthin and lutein concentrations of men ( $\bigcirc$ and $\longrightarrow$ ) and women $(\bigcirc$ and ----$)$ according to their respective mean daily dietary intake as well as daily number of fruit and vegetable (FAV) servings. Values are means and standard deviations. Data were adjusted for age. lut + zea, Lutein + zeaxanthin.
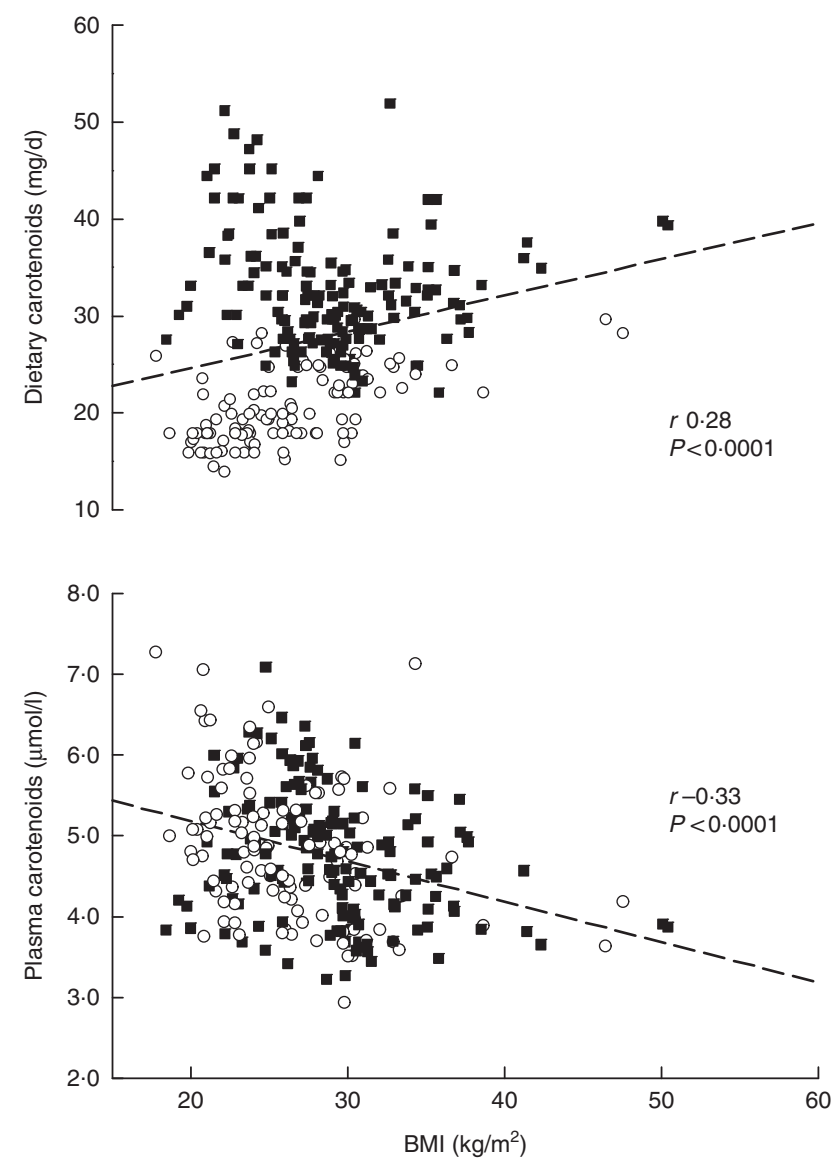

Fig. 4. Associations between BMI of the 264 participants and their total dietary carotenoid intakes (top panel) and total plasma carotenoid concentrations (bottom panel). $\square$, Men; $O$, women. concentrations and FAV intake. Our results are, however, in line with those suggesting that plasma $\beta$-carotene concentration may not be a suitable marker of FAV intake as it may be affected by specific dietary patterns ${ }^{(38)}$.

Lycopene, a carotenoid highly abundant in tomatoes ${ }^{(11)}$, has attracted much attention in recent years because of its potential benefits against chronic diseases ${ }^{(39)}$. There is a positive relationship between circulating lycopene concentrations and FAV consumption ${ }^{(31)}$, although this association was not reported by all ${ }^{(14,29)}$. Lycopene is, however, recognised as a better biomarker of tomato (whole and processed) consumption than of mixed FAV intake ${ }^{(10)}$. In the present study, plasma lycopene concentrations were positively associated with dietary lycopene intake but also negatively correlated to the daily number of FAV servings consumed by the participants. This could be due to the fact that the diets with the highest lycopene contents (diets 1 and 4) were not the ones with the highest number of daily FAV servings consumed. When we further investigated foods that contributed to dietary lycopene intake within the context of our diet-controlled interventions, we found that starchy vegetables (potatoes and maize) best predicted dietary lycopene intake (data not shown). Starchy vegetables are not a source of lycopene, but they were used in the preparation of meals (e.g. mixed with ground beef) consumed in the context of diets 1 and 4 and diets that were accompanied by ketchup and chili sauce, which are potent sources of lycopene ${ }^{(11,40)}$. Thus, our results suggest that circulating lycopene concentrations may not be the best-suited biomarker of mixed FAV intake in diets where processed tomato products are also consumed.

We further investigated potential sex differences in circulating carotenoid concentrations, and found that plasma 
lutein concentrations were significantly higher in women compared with men regardless of the number of daily FAV servings consumed. We also noted that associations between plasma $\beta$-cryptoxanthin and FAV intake were different between men and women, as reflected by a significant sex $\times$ daily FAV servings interaction determining circulating $\beta$-cryptoxanthin concentrations. Specifically, women showed a proportionally larger increase in plasma $\beta$-cryptoxanthin concentrations with increasing FAV intake compared with men. Such sex differences in circulating lutein and $\beta$-cryptoxanthin concentrations have already been reported ${ }^{(13,41)}$, although the explanation for such a phenomenon remains to be elucidated. Previous observations have, however, suggested that body size may interfere with the relationship between dietary carotenoid intake and plasma concentration, with overweight individuals showing lower circulating carotenoid concentrations compared with non-obese subjects ${ }^{(12)}$ probably because of the accumulation of carotenoids within adipose tissue ${ }^{(42)}$. In the present study, we found a negative association between BMI and total plasma carotenoid concentrations, which may partly explain the observed sex difference in total plasma carotenoid concentrations, as women who showed lower mean BMI compared with men were also those showing higher total plasma carotenoid concentrations. This reinforces the notion that besides potential differences in absorption and/or metabolism of dietary carotenoids obesity status must also be considered when comparing and interpreting the physiological and nutritional relevance of circulating carotenoid concentrations.

Strengths of our study include the use of data from a fairly large sample of men and women enrolled in fully controlled feeding studies, allowing us to examine the relationship of circulating biomarkers of consumption with the actual number of daily FAV servings consumed by participants, whereas most studies looking at carotenoids as biomarkers of FAV consumption have relied on data collected through selfreported dietary assessment tools ${ }^{(13,14,32,34,36,37)}$. During the preparation of the present article, a meta-analysis was published $^{(43)}$ using data from twelve diet-controlled intervention studies including 526 European men and women in whom circulating carotenoid measures were available (largest sample size for $\beta$-carotene $(n$ 316) and smallest sample size for lutein + zeaxanthin $(n$ 35)). Studies considered for this metaanalysis were interventions in which all foods and drinks or all FAV consumed were provided to the participants for periods varying from 7 to $90 \mathrm{~d}$. Souverein et $a l .{ }^{(43)}$ reported that circulating $\beta$-cryptoxanthin and lutein concentrations were significantly associated with FAV intake, which is in line with the results we have reported in this study. However, contrary to what we found, plasma/serum $\alpha$-carotene, $\beta$-carotene and lycopene concentrations were also positively associated with FAV intake ${ }^{(43)}$. When taken together, our results and those of Souverein et al. ${ }^{(43)}$ suggest that circulating $\beta$-cryptoxanthin and lutein concentrations may be more robust biomarkers of mixed FAV intake than $\alpha$-carotene, $\beta$-carotene and lycopene, which in turn could be more sensible to the presence of specific FAV categories (or by-products) in the diet.

The present study also has limitations that must be acknowledged. First, dietary interventions that were compiled in the present analyses were not primarily designed to identify biomarkers of FAV consumption. For instance, in our analyses, the lowest FAV intake of the participants was about 7 servings/d - a value that actually falls within the range of the recommended FAV intake, that is, 7-10 FAV servings/d ${ }^{(44)}$. However, it must be kept in mind that a large number of individuals do not meet nutritional recommendations and especially those for FAV. Indeed, $>60 \%$ of Canadians aged 12 years or older consume $<5 \mathrm{FAV}$ servings $/ \mathrm{d}^{(45)}$. In the USA, $13.1 \%$ of adults reportedly meet fruit intake recommendations and $8.9 \%$ meet those for vegetables ${ }^{(46)}$, whereas in Europe a majority of people do not reach the WHO daily recommendation for FAV consumption of $\geq 400 \mathrm{~g} / \mathrm{d}^{(47)}$. In the studies we used for the present analysis, FAV consumption was well above the WHO threshold for all participants. Therefore, it would have been interesting to have been able to include studies with a lower range of FAV intakes not only in an attempt to identify threshold values for circulating biomarkers such as plasma carotenoid concentrations associated with the ideal number of FAV servings needed to reduce chronic disease risk, but also to verify to what extent plasma carotenoids also correlate with lower daily FAV intake. Second, there are variations in the FAV contents of the six dietary interventions used in our analyses, which could impact the validity of our observations as the proportion of carotenoids that are consumed likely differ between diets. On the other hand, this variety in FAV provided and consumed could also been seen as a strength as it may better reflect the mixed FAV intakes generally observed in the population. Third, it has been suggested that plasma carotenoids may be more powerful markers of the changes in the consumption of FAV. For our analyses, we compared post-intervention plasma carotenoid concentrations and FAV intakes rather than their respective changes over the course of the interventions, which may have yielded stronger relationships. Finally, our study does not take into account potential metabolic or genetic characteristics of subjects or even seasonal variations in the carotenoid content of foods, which most likely affect carotenoid metabolism and circulating concentrations.

In summary, the present study shows that plasma $\beta$-cryptoxanthin and lutein concentrations are reliable biomarkers of mixed FAV consumption. Our results also provide us with an insight on the importance of sex as a determinant of circulating plasma carotenoid concentrations, especially $\beta$-cryptoxanthin and lutein. The reliability of using circulating $\beta$-cryptoxanthin, lutein or other carotenoid concentrations as biomarkers of mixed FAV consumption and their relevance in the validation of self-reporting dietary assessment methods in different populations (adults, adolescents, elderly, pregnant women, etc.) will need to be examined in future studies. In addition, the determinants of sex difference in the response of plasma $\beta$-cryptoxanthin and lutein concentrations to increased FAV consumption will need to be further investigated as well as how this affects the health benefits of FAV in chronic disease conditions.

\section{Acknowledgements}

The authors acknowledge the contribution of Pascal Dubé of the Institute of Nutrition and Functional Foods for the 
measurements of plasma carotenoid concentrations. The authors also acknowledge the contributions of nurses and other research professionals as well as subjects who participated in the dietary interventions, without whom no clinical research would be possible.

Studies were made possible by grants from the Canadian Institutes of Health Research (MOP-68866, MOP-84568 and FHG-129921), the Natural Sciences and Engineering Research Council of Canada, the Dairy Farmers of Canada, the Canadian Dairy Commission, Novalait Inc., Dairy Australia, Agriculture and Agri-Food Canada, the Canola Council of Canada, the Flax Council of Canada, Dow Agrosciences, the Western Grains Research Foundation of Canada, the National Center for Research Resources (UL1 RR033184) and the National Center for Advancing Translational Sciences (UL1 TR000127). Funders of the studies had no role in the design, conduct, management, data collection, analysis or writing or review of the present article.

The authors' responsibilities were as follows: C. C., S. L., M.-C. V., P. C. and B. L.: research design; P. C.: medical supervision of study participants; C. C., S. L., M.-C. V., P. C. and B. L.: data acquisition; P. C.: medical supervision of study participants; C. C.: analysis and interpretation of the data and drafting of the manuscript; M.-C. V., P. C., S. L. and B. L.: critical revision of the manuscript for important intellectual content. All the authors read and approved the final manuscript.

C. C. has received research funding from the Canadian Cranberry Growers Coalition and McCormick Science Institute and received honoraria or travel expense reimbursements from the Berry Health Benefits Symposium, Ocean Spray Cranberries Inc. and Mott's Canada. C. C. and B. L. have received research funding from Atrium Innovations. B. L. has received research funding from the Danone Institute and honoraria from Unilever, Danone and the Dairy Farmers of Canada. B. L. is Chair in Nutrition and Cardiovascular Health, supported in part by Provigo/Loblaws. B. L. and P. C. have received research grants from the Dairy Farmers of Canada and Dairy Australia.

\section{Supplementary material}

For supplementary material/s referred to in this article, please visit http://dx.doi.org/10.1017/S0007114516003056

\section{References}

1. Crowe FL, Roddam AW, Key TJ, et al. (2011) Fruit and vegetable intake and mortality from ischaemic heart disease: results from the European Prospective Investigation into Cancer and Nutrition (EPIC)-Heart study. Eur Heart J 32, 1235-1243.

2. Yusuf S, Hawken S, Ounpuu S, et al. (2004) Effect of potentially modifiable risk factors associated with myocardial infarction in 52 countries (the INTERHEART study): case-control study. Lancet 364, 937-952.

3. Carter P, Gray LJ, Troughton J, et al. (2010) Fruit and vegetable intake and incidence of type 2 diabetes mellitus: systematic review and meta-analysis. BMJ 341, c4229.

4. World Cancer Research Fund \& American Institute for Cancer Research (AICR) (2007) Food, Nutrition, Physical Activity, and the Prevention of Cancer: A Global Perspective. Washington, DC: AICR.
5. Subar AF (2004) Developing dietary assessment tools. J Am Diet Assoc 104, 769-770.

6. Archer E \& Blair SN (2015) Implausible data, false memories, and the status quo in dietary assessment. Adv Nutr 6, 229-230.

7. Miller TM, Abdel-Maksoud MF, Crane LA, et al. (2008) Effects of social approval bias on self-reported fruit and vegetable consumption: a randomized controlled trial. Nutr J 7, 18.

8. Westerterp KR \& Goris AH (2002) Validity of the assessment of dietary intake: problems of misreporting. Curr Opin Clin Nutr Metab Care 5, 489-493.

9. Livingstone MB \& Black AE (2003) Markers of the validity of reported energy intake. J Nutr 133, Suppl. 3, 895S-920S.

10. Baldrick FR, Woodside JV, Elborn JS, et al. (2011) Biomarkers of fruit and vegetable intake in human intervention studies: a systematic review. Crit Rev Food Sci Nutr 51, 795-815.

11. Maiani G, Caston MJ, Catasta G, et al. (2009) Carotenoids: actual knowledge on food sources, intakes, stability and bioavailability and their protective role in humans. Mol Nutr Food Res 53, Suppl. 2, S194-S218.

12. Vioque J, Weinbrenner T, Asensio L, et al. (2007) Plasma concentrations of carotenoids and vitamin $\mathrm{C}$ are better correlated with dietary intake in normal weight than overweight and obese elderly subjects. BrJ Nutr 97, 977-986.

13. Jansen MC, Van Kappel AL, Ocke MC, et al. (2004) Plasma carotenoid levels in Dutch men and women, and the relation with vegetable and fruit consumption. Eur J Clin Nutr 58, 1386-1395.

14. Carlsen MH, Karlsen A, Lillegaard IT, et al. (2011) Relative validity of fruit and vegetable intake estimated from an FFQ, using carotenoid and flavonoid biomarkers and the method of triads. Br J Nutr 105, 1530-1538.

15. Crispim SP, Geelen A, Souverein OW, et al. (2011) Biomarkerbased evaluation of two 24-h recalls for comparing usual fish, fruit and vegetable intakes across European centers in the EFCOVAL Study. Eur J Clin Nutr 65, Suppl. 1, S38-S47.

16. Kristal AR, Vizenor NC, Patterson RE, et al. (2000) Precision and bias of food frequency-based measures of fruit and vegetable intakes. Cancer Epidemiol Biomarkers Prev 9, 939-944.

17. Bogers RP, Dagnelie PC, Westerterp KR, et al. (2003) Using a correction factor to correct for overreporting in a foodfrequency questionnaire does not improve biomarkerassessed validity of estimates for fruit and vegetable consumption. J Nutr 133, 1213-1219.

18. Bogers RP, Van Assema P, Kester AD, et al. (2004) Reproducibility, validity, and responsiveness to change of a short questionnaire for measuring fruit and vegetable intake. Am J Epidemiol 159, 900-909.

19. Bédard A, Riverin M, Dodin S, et al. (2012) Sex differences in the impact of the Mediterranean diet on cardiovascular risk profile. Br J Nutr 108, 1428-1434.

20. Richard C, Couture P, Desroches S, et al. (2012) Effect of the Mediterranean diet with and without weight loss on surrogate markers of cholesterol homeostasis in men with the metabolic syndrome. Br J Nutr 107, 705-711.

21. Jones PJ, Senanayake VK, Pu S, et al. (2014) DHA-enriched high-oleic acid canola oil improves lipid profile and lowers predicted cardiovascular disease risk in the canola oil multicenter randomized controlled trial. Am J Clin Nutr 100, 88-97.

22. Labonté ME, Couture P, Paquin P, et al. (2011) Comparison of the impact of trans fatty acids from ruminant and industrial sources on surrogate markers of cholesterol homeostasis in healthy men. Mol Nutr Food Res 55, Suppl. 2, S241-S247. 
23. Lacroix E, Charest A, Cyr A, et al. (2012) Randomized controlled study of the effect of a butter naturally enriched in trans fatty acids on blood lipids in healthy women. Am J Clin Nutr 95, 318-325.

24. Gray-Donald K, Jacobs-Starkey L \& Johnson-Down L (2000) Food habits of Canadians: reduction in fat intake over a generation. Can J Public Health 91, 381-385.

25. Willett WC, Sacks F, Trichopoulou A, et al. (1995) Mediterranean diet pyramid: a cultural model for healthy eating. Am J Clin Nutr 61, 1402S-1406S.

26. Saura-Calixto F \& Goni I (2009) Definition of the Mediterranean diet based on bioactive compounds. Crit Rev Food Sci Nutr 49, 145-152.

27. van der Kooy K \& Seidell JC (1993) Techniques for the measurement of visceral fat: a practical guide. Int J Obes Relat Metab Disord 17, 187-196.

28. Gleize B, Steib M, Andre M, et al. (2012) Simple and fast HPLC method for simultaneous determination of retinol, tocopherols, coenzyme Q(10) and carotenoids in complex samples. Food Chem 134, 2560-2564.

29. Daniels JA, Mulligan C, McCance D, et al. (2014) A randomised controlled trial of increasing fruit and vegetable intake and how this influences the carotenoid concentration and activities of PON-1 and LCAT in HDL from subjects with type 2 diabetes. Cardiovasc Diabetol 13, 16.

30. Macdonald HM, Hardcastle AC, Duthie GG, et al. (2009) Changes in vitamin biomarkers during a 2-year intervention trial involving increased fruit and vegetable consumption by free-living volunteers. Br J Nutr 102, 1477-1486.

31. Crane TE, Kubota C, West JL, et al. (2011) Increasing the vegetable intake dose is associated with a rise in plasma carotenoids without modifying oxidative stress or inflammation in overweight or obese postmenopausal women. J Nutr 141, 1827-1833.

32. Campbell DR, Gross MD, Martini MC, et al. (1994) Plasma carotenoids as biomarkers of vegetable and fruit intake. Cancer Epidemiol Biomarkers Prev 3, 493-500.

33. Block G, Norkus E, Hudes M, et al. (2001) Which plasma antioxidants are most related to fruit and vegetable consumption? Am J Epidemiol 154, 1113-1118.

34. Brevik A, Andersen LF, Karlsen A, et al. (2004) Six carotenoids in plasma used to assess recommended intake of fruits and vegetables in a controlled feeding study. Eur J Clin Nutr 58, $1166-1173$.
35. Polsinelli ML, Rock CL, Henderson SA, et al. (1998) Plasma carotenoids as biomarkers of fruit and vegetable servings in women. J Am Diet Assoc 98, 194-196.

36. Al-Delaimy WK, Ferrari P, Slimani N, et al. (2005) Plasma carotenoids as biomarkers of intake of fruits and vegetables: individual-level correlations in the European Prospective Investigation into Cancer and Nutrition (EPIC). Eur J Clin Nutr 59, 1387-1396.

37. van Kappel AL, Steghens JP, Zeleniuch-Jacquotte A, et al. (2001) Serum carotenoids as biomarkers of fruit and vegetable consumption in the New York Women's Health Study. Public Health Nutr 4, 829-835.

38. Garcia AL, Mohan R, Koebnick C, et al. (2010) Plasma betacarotene is not a suitable biomarker of fruit and vegetable intake in German subjects with a long-term high consumption of fruits and vegetables. Ann Nutr Metabol 56, 23-30.

39. Wang XD (2012) Lycopene metabolism and its biological significance. Am J Clin Nutr 96, 1214S-1222S.

40. Clinton SK (1998) Lycopene: chemistry, biology, and implications for human health and disease. Nutr Rev 56, 35-51.

41. Olmedilla B, Granado F, Blanco I, et al. (1994) Seasonal and sex-related variations in six serum carotenoids, retinol, and alpha-tocopherol. Am J Clin Nutr 60, 106-110.

42. El-Sohemy A, Baylin A, Kabagambe E, et al. (2002) Individual carotenoid concentrations in adipose tissue and plasma as biomarkers of dietary intake. Am J Clin Nutr 76, 172-179.

43. Souverein OW, de Vries JH, Freese R, et al. (2015) Prediction of fruit and vegetable intake from biomarkers using individual participant data of diet-controlled intervention studies. Br J Nutr 113, 1396-1409.

44. Health Canada (2011) Eating well with Canada's food guide. http://www.hc-sc.gc.ca/fn-an/alt_formats/hpfb-dgpsa/ pdf/food-guide-aliment/view_eatwell_vue_bienmang-eng.pdf (accessed December 2015).

45. Health Canada (2015) Fruit and vegetable consumption. Canadian Community Health Survey 2014. http://www. statcan.gc.ca/pub/82-625-x/2015001/article/14182-eng.htm (accessed May 2016).

46. Moore LV \& Thompson ET (2015) Adults meeting fruit and vegetable intake recommendations - United States, 2013. Morb Mortal Wkly Rep 64, 709-713.

47. World Health Organization (2008) WHO European Action Plan for Food and Nutrition 2007-2012. Copenhagen, Denmark: WHO. 